\title{
Quantitative trait loci mapping of pubescence density and flowering time of insect-resistant soybean (Glycine max L. Merr.)
}

\author{
Kunihiko Komatsu ${ }^{1}$, Shiori Okuda ${ }^{1}$, Masakazu Takahashi ${ }^{1}$, Ryoichi Matsunaga ${ }^{2}$ and Yoshinori Nakazawa ${ }^{1}$ \\ ${ }^{l}$ National Agriculture and Food Research Organization, National Agricultural Research Center \\ for Kyushu Okinawa Region, Koshi, Kumamoto, Japan. \\ ${ }^{2}$ Japan International Research Center for Agricultural Science, Tsukuba, Ibaraki, Japan.
}

\begin{abstract}
Analysis of antibiosis resistance to common cutworm (Spodoptera litura Fabricius) in soybean (Glycine max (L.) Merr.) has progressed significantly, but the immediate cause remains unknown. We performed quantitative trait loci (QTL) analysis of pubescence density and plant development stage because these factors are assumed to be the immediate cause of resistance to cutworm. The QTLs for pubescence appeared to be identical to the previously detected the Pd1 and Ps loci controlling pubescence density. We found no candidate loci for flowering time QTLs, although one could be identical to the gene governing the long-juvenile trait or to the E6 loci controlling maturity. None of the QTLs overlapped with the QTLs for antibiosis resistance.
\end{abstract}

Key words: Glycine max, hair, maturity, pest resistance, QTL, SSR.

Received: May 16, 2006; Accepted: January 31, 2006.

To reduce damage to soybean (Glycine max (L.) Merr.) plants caused by herbivorous insects, especially the common cutworm (Spodoptera litura Fabricius, Lepidoptera, Noctuidae) (CCW), breeding programs to develop pest-resistant soybean cultivars have been conducted in Japan. We reported that the soybean cultivar Himeshirazu exhibits effective antibiosis resistance to $S$. litura (Komatsu et al., 2004). We genetically analyzed this resistance using DNA markers and recognized two quantitative trait loci (QTL) for antibiosis resistance to $S$. litura located on $G$. max linkage group $\mathrm{M}$ which we named $C C W-1$ and $C C W-2$ and which explain $28 \%$ and $16 \%$, respectively, of phenotypic variance of antibiosis (Komatsu et al., 2005).

The genetics of the resistance has been studied (Komatsu et al., 2005) but its immediate cause remains unknown, although pubescence density is a possible factor. Lambert et al. (1992) reported that pupal weight and duration from hatching till pupation of the corn (maize) earworm (Helicoverpa zea Boddie) were affected by pubescence density when near-isogenic soybean lines differing only in pubescence density were used as diet. It is necessary to clarify the relationship between the resistance of the Himeshirazu soybean cultivar to $S$. litura and pubescence density for better identification of the mechanisms of resistance to $S$. litura.

Send correspondence to Kunihiko Komatsu. National Agricultural Research Center for Kyushu Okinawa Region, 2421 Suya, Koshi, 861-1192 Kumamoto, Japan. E-mail: kkomatsu@ affrc.go.jp.
The plant development stage is also a possible factor in resistance. Nault et al. (1992) reported that mortality of Pseudoplusia includens (Walker) was lower in larvae reared on soybean at the plant reproductive phase than in larvae reared at the vegetative phase. Within the vegetative phase, plant age affected the growth rate of $P$. includens larvae (Reynolds and Smith, 1985). The Himeshirazu cultivar matures late (maturity group VIII), later than susceptible, mid-maturity cultivars. This later development could contribute to antibiosis resistance, but this would be difficult to determine because it is difficult to synchronize the development stage of all the materials needed for the bioassay. On the other hand, the late maturity of the Himeshirazu cultivar could be an obstacle for the development of elite pestresistant cultivars, because it is too late for the cultivation program followed in Japan. If late maturity confers resistance, it might be difficult to develop elite pest-resistant cultivars. We would also face the same difficulty if the genes for resistance and maturity are tightly linked, even though maturity is not the cause of the resistance.

Genetic studies on soybean maturity have revealed the positions of some maturity genes and QTLs (Harada and Xia, 2004), but the materials studied were relatively early-maturing cultivars so the effects of the genes and QTLs on the genetic control of late maturity is still unclear. We conducted genetic analysis of the factors involved in the maturity of the Himeshirazu cultivar with the objectives of detecting the positions and effects of QTLs for pubes- 
cence density and maturity of pest-resistant soybean and also to clarify the effects of pubescence density and maturity on pest resistance.

The Japanese soybean cultivars Himeshirazu and Fukuyutaka were crossed, with the Fukuyutaka cultivar as the maternal parent, and their $F_{1}$ and $F_{2}$ descendants used in this study. The Himeshirazu cultivar exhibits effective resistance to $S$. litura (Komatsu et al., 2004) plus late maturity (MG VIII), its pubescence being denser than that of the Fukuyutaka cultivar which is a leading cultivar in southwestern Japan but is susceptible to $S$. litura (Komatsu et al., 2004) and belongs to MG VI. The $\mathrm{F}_{2}$ population consisted of 143 plants. Seeds of all materials were sown in potting compost on 31 May 2001 and transplanted into the experimental field 2 weeks later. The $\mathrm{F}_{2}$ seedlings were numbered at random and transplanted into five rows, each with 35 plants. The distance between plants in a row was $30 \mathrm{~cm}$, and the distance between rows was $75 \mathrm{~cm}$. The parents and $F_{1}$ offspring were also planted into the same experimental field. Plants were grown in the experimental field of the $\mathrm{Na}$ tional Agricultural Research Center for Kyushu Okinawa Region, Japan at $32^{\circ} 52^{\prime} \mathrm{N}, 130^{\circ} 44^{\prime} \mathrm{E}$ and the traits were also investigated there. The same plant materials were used for genetic analysis of resistance to $S$. litura (Komatsu et al., 2004, 2005).

Pubescence density was evaluated as the number of hairs per $10 \mathrm{~mm}^{2}$ of the underside of the leaf. Four leaflets that had just expanded fully were collected from each plant and a $10 \mathrm{~mm}^{2}$ area of the underside of each leaflet was displayed on a TV screen connected to a stereomicroscope equipped with an ARGUS-10 image processor (Hamamatsu Photonics K.K., Hamamatsu, Japan) and the hairs counted. The area was chosen to avoid any veins or scars. The mean of four density evaluations was used as the index of pubescence density. The flowering time of each plant was expressed as the days from sowing to flowering. Flowering was checked at least every 2 days from 23 July (54th day from sowing) until 25 August (87th day from sowing) when all plants had flowered.

For QTL analysis we constructed a linkage map, reported in detail by Komatsu et al. (2005). As mapping loci we used the phenotype $T$ locus governing pubescence color (Woodworth, 1921) and 146 simple sequence repeat (SSR) loci, 139 of which were reported by Cregan et al. (1999) or published in Soybase (http://soybase.org). The other seven were developed by Prof. K. Harada at Chiba University, Japan (personal communication). Total DNA was extracted from leaves by the method of Doyle and Doyle (1990) and the PCR amplification conditions and electrophoresis procedure were as previously reported by Komatsu et al. (2004, 2005). We used MAPMAKER/EXP 3.0b (Lander et al., 1987) to construct a linkage map containing 23 linkage groups spanning 2270.4 centimorgan (cM) (Komatsu et al., 2005). The positions and effects of the QTLs were calculated using the composite interval mapping (CIM) method
(Zeng, 1993, 1994) and the Windows QTL Cartographer 1.21 software. The LOD score threshold for QTL detection was estimated by means of a permutation test (Churchill and Doerge, 1994) with 1000 permutations for each trait. The threshold level of the logarithm of the odds (LOD) score was 3.52 for pubescence density and 3.68 for flowering time, equivalent to a $5 \%$ genome-wise Type I error rate for each trait.

The pubescence densities per $10 \mathrm{~mm}^{2}$ of leaf underside were 30.5 in Fukuyutaka, 57.6 in Himeshirazu, and 49.7 in the $F_{1}$. In the histogram shown in Figure 1a the smooth distribution of the segregating $\left(\mathrm{F}_{2}\right)$ population indicates that no gene had a prominent effect on pubescence density, with obvious transgressive segregation also being shown in the histogram. Four plants in the population were excluded from the analysis because they possessed insufficient leaves suitable for analysis. The QTL analysis of the segregating data using the CIM method detected two QTLs located on linkage groups $\mathrm{D} 1 \mathrm{a}+\mathrm{Q}$ and $\mathrm{H}$ (labeled $\mathrm{P}$ in
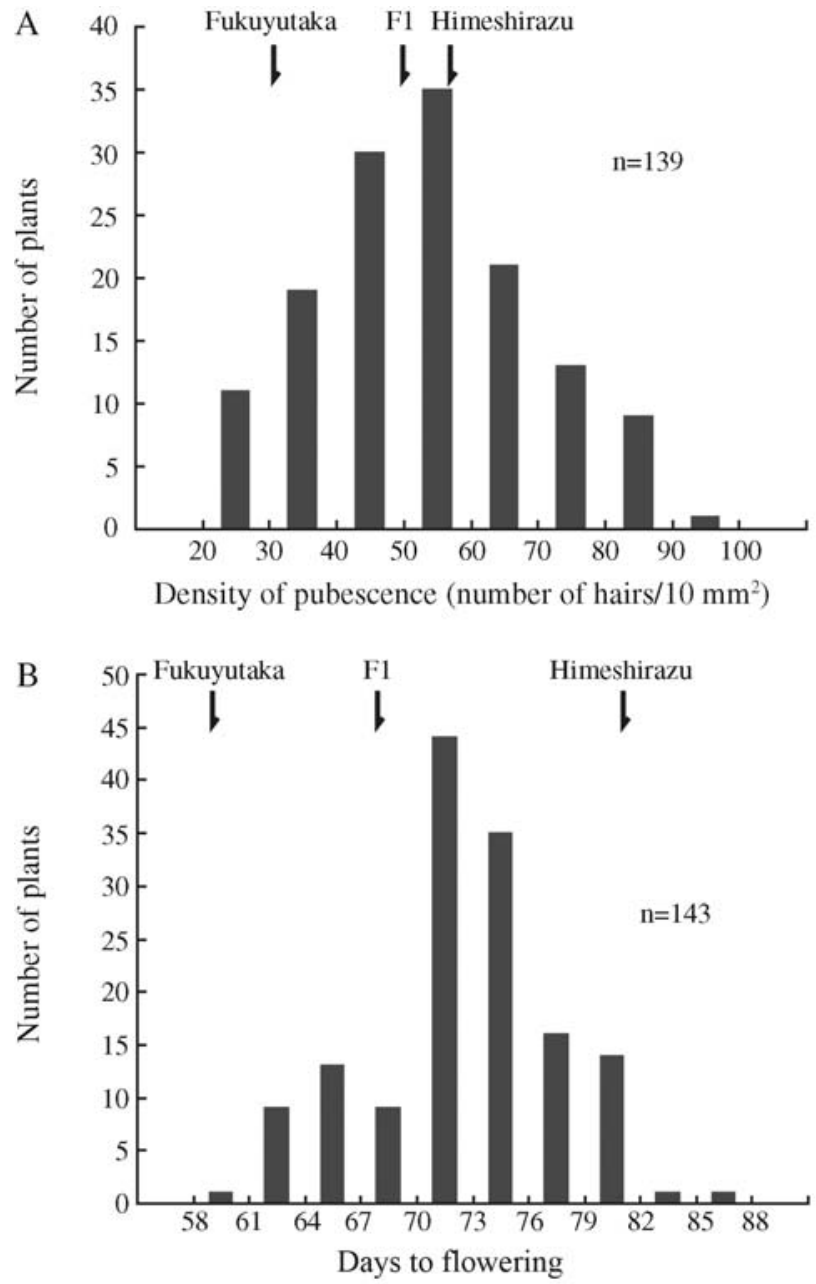

Figure 1 - Distribution of pubescence density and days to flowering in the $\mathrm{F}_{2}$ segregating population derived from a cross between the Fukuyutaka and Himeshirazu cultivars. (A) The number of hairs in $10 \mathrm{~mm}^{2}$ of leaf underside. Four plants were excluded from analysis because they did not possess sufficient leaves for analysis. (B) Days to flowering from sowing. 
Figure 2), the LOD scores and effects of which being shown in Table 1. The effects indicated that the Himeshirazu allele on linkage group D1a+Q and the Fukuyutaka allele on linkage group $\mathrm{H}$ increased the pubescence density. However, neither position overlapped with QTL for antibiosis resistance (labeled $\mathrm{S}$ in Figure 2).

Four genes related to pubescence density have been reported (Palmer and Kilen, 1987), three of which are $P d 1$, $P S$, and $P 1$ which are located on linkage groups $\mathrm{D} 1 \mathrm{a}+\mathrm{Q}, \mathrm{H}$ and $\mathrm{K}$ respectively (Cregan et al., 1999). Of the alleles which we detected on linkage groups $\mathrm{D} 1 \mathrm{a}+\mathrm{Q}$ and $\mathrm{H}$ the allele for dense pubescence was estimated to be relatively dominant, this being consistent with the fact that the $F_{1}$ plants had denser pubescence than the Fukuyutaka parent and were thus closer to that of the Himeshirazu dense pubescence parent. These QTLs lie close to the $P d l$ and $P s$, respectively (Cregan et al., 1999) and could thus be identical to them. The QTL on linkage group $\mathrm{H}$ had a larger additive effect and $r^{2}$ value (Table 1), although the allele that increased the pubescence density is derived from the
Fukuyutaka cultivar. This fact is consistent with the transgressive segregation detected in the $\mathrm{F}_{2}$ population. But it is odd that the allele most effective at increasing pubescence density originated in the sparsely pubescent parent. In addition, the dense pubescence allele is nearly dominant, although a sparse or semi-sparse allele is dominant at the candidate locus Ps (Bernard and Singh, 1969; Bernard, 1975). The Fukuyutaka allele may be new, or of course it is also possible that the QTL is different from the Ps locus. The positions of the two pubescence QTLs do not overlap with those of the antibiosis QTLs on linkage group M, indicating that pubescence density has little influence on the antibiosis resistance of the Himeshirazu cultivar. In addition, the pubescence QTLs did not overlap with the QTLs of another mode of resistance (Rector et al., 1998, 1999), called antixenosis or non-preference (Kogan and Ortman, 1978), on linkage groups C2, D1b+W, F, H and M. The pubescence QTL on LG-H belonged to the same linkage group as an antixenosis QTL but their linkage distance was over $68 \mathrm{cM}$ (data not shown). It is difficult to find a positive
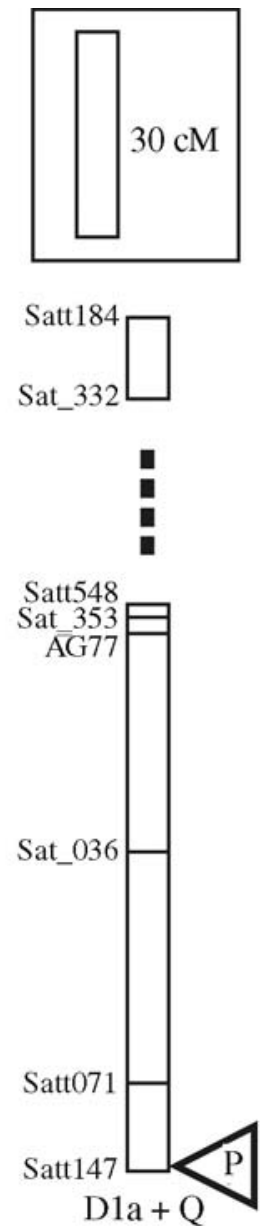

$\mathrm{D} 1 \mathrm{a}+\mathrm{Q}$

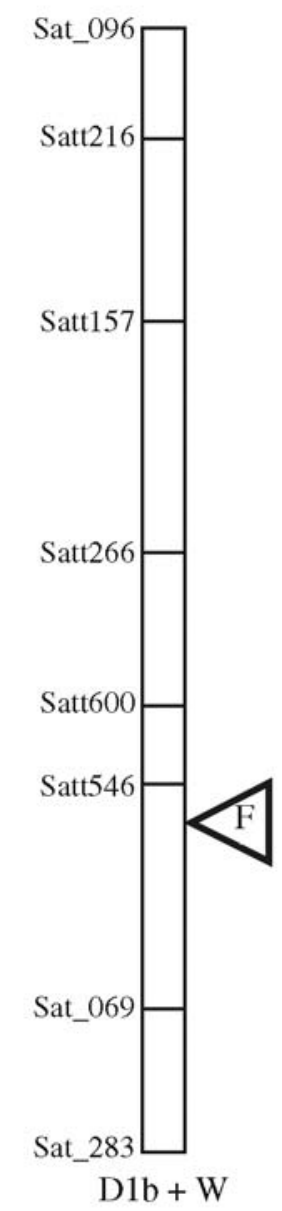

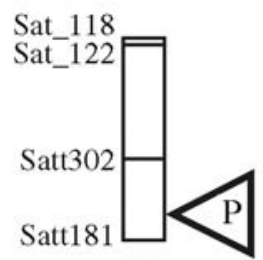

$\mathrm{H}$
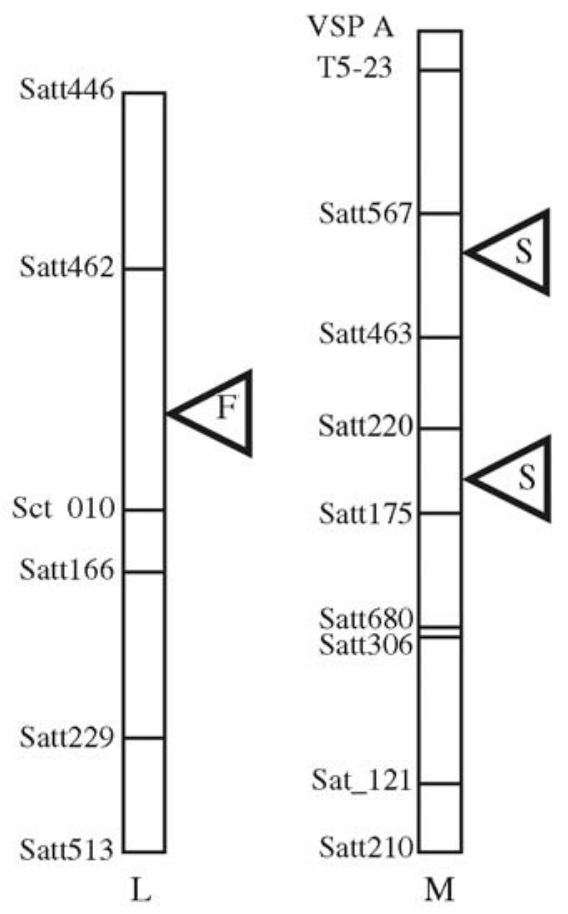

Figure 2 - Location of the quantitative trait loci (QTLs) on linkage groups (LGs) (vertical bars). Labels to the left of the bars indicate marker names. The dotted lines in LG D1a+Q indicate breaking off of linkage in our map. Triangles point to the peak of the logarithm of odds curve for each QTL. 'P' and 'F' indicate pubescence density and days to flowering. 'S' indicates the two QTLs for antibiosis resistance to common cutworm (Komatsu et al., 2005). The name of each LG is based on Cregan et al. (1999). 
Table 1 - Positions and effects of quantitative trait loci (QTL) detected in the $\mathrm{F}_{2}$ progeny of the Fukuyutaka x Himeshirazu cross. The Logarithm of odds (LOD) score threshold for QTL detection was 3.52 for pubescence density and 3.68 for flowering time. For the additive effect if the locus is fixed with the maternal Fukuyutaka parent allele the trait value increases by the indicated value from the intermediate value between the parents. For the dominance effect if the locus is heterozygous the trait value increases by the indicated value from the intermediate value between the parents. The $r^{2}$ value represents the proportion of variance explained by the locus over the total phenotypic variance.

\begin{tabular}{lcccccc}
\hline Trait & Linkage group & Closest marker & LOD score & Additive effect & Dominance effect & $\mathrm{r}^{2}$ value \\
\hline Pubescence density & D1a+Q & Satt147 & 5.0 & -8.01 & 1.62 & 0.135 \\
& $\mathrm{H}$ & Satt181 & 5.0 & 8.22 & 4.06 & 0.163 \\
Days to flowering & D1b+W & Satt546 & 3.7 & 3.46 & -1.94 & 0.152 \\
& $\mathrm{~L}$ & Sct_010 & 4.9 & -3.49 & 1.12 & 0.238 \\
\hline
\end{tabular}

relationship between the pubescence density and antixenosis resistance in the results of this study and the reports of Rector et al. (1998, 1999). On the other hand, our results indicate that it is possible to develop densely pubescent or sparsely pubescent CCW-resistant soybean cultivars. Lambert et al. (1992) reported that dense pubescence promotes the oviposition of lepidopteran insects. Sparsely pubescent cultivars with antibiosis resistance might contribute more effectively to the reduction of loss from S. litura.

For each plant we recorded the number of days from sowing to anthesis and found that the Fukuyutaka cultivar flowered at day 59, the Himeshirazu cultivar at day 81 and the $F_{1}$ at day 68 . A histogram of the segregating $F_{2}$ population is shown in Figure 1b. The QTL analysis for days to anthesis indicated that the regulation of flowering time is affected by two QTLs located on linkage groups D1b+W and L (labeled F in Figure 2), their positions and effects being shown in Table 1. The Fukuyutaka allele of the QTL on linkage group $\mathrm{D} 1 \mathrm{~b}+\mathrm{W}$ delays flowering while the Himeshirazu allele for early flowering is nearly dominant. The positive value of the additive effect indicates that the maternal Fukuyutaka allele restrains anthesis, while the negative value of the dominance effect indicates that the allele for early flowering is dominant. Conversely, the Himeshirazu allele of the QTL on linkage group L delays flowering. The positions of the two QTLs did not overlap with those of the QTLs for antibiosis (labeled S in Figure 2).

One of the qualitative soybean genes for flowering time is the E3 locus located on linkage group L (Molnar et al., 2003) and, interestingly, we detected a QTL on linkage group L but we feel that these loci are distinct from each other, although an allelism test may be required to confirm their relationship. Molnar et al. (2003) reported that the E3 locus is located near the SSR locus Satt229 and Funatsuki et al. (2005) also estimated the position of $E 3$ to be near the Satt229 locus. In contrast, the QTL detected by us analysis lies $54.0 \mathrm{cM}$ from Satt229 (data not shown) so it is difficult to regard them as the same locus. No candidate locus could be detected for the other QTL on linkage group D1b+W but some interesting sites were identified. The Himeshirazu cultivar early-flowering allele was nearly dominant, even though the ordinary early alleles in conventional maturity genes (e.g. E1 to E5 in soybean) are recessive. It is interesting to note that the nearly dominant early-flowering allele originated in the late-maturing Himeshirazu cultivar while the late-flowering allele was from the earlier-flowering Fukuyutaka cultivar. The $J$ allele, conferring the long-juvenile (LJ) trait, has been reported as a dominant allele for early flowering (Ray et al., 1995). Carpentieri-Pípolo et al. (2000) reported that three genes govern the LJ trait and although they did not analyze allelism they did find that recessive alleles are required for the LJ trait, implying that all dominant alleles lead to early-flowering. It is still unclear, however, if the Fukuyutaka and Himeshirazu cultivars have the LJ trait or not and it is also uncertain whether the three genes conferring LJ influence flowering time or not under a non-LJ genetic background. However, if these genes have influence even under a non-LJ background, it is not strange that a dominant allele for early-flowering originated in the late-maturing Himeshirazu cultivar. Another gene for flowering time (EG) has been reported, of which the dominant allele induces earlier flowering (Bonato and Vello, 1999). The E6 gene was detected in a genetic analysis using a relatively late-maturing (MG-VI) cultivar, and its natural mutants cause later flowering and maturity. Our result is not strange if $E 6$ is one of the causes of the maturity difference between the relatively late-maturing Fukuyutaka cultivar and the even later-maturing Himeshirazu cultivar. More discussion of the E6 gene and the QTL on linkage group $\mathrm{D} 1 \mathrm{~b}+\mathrm{W}$ is prevented by lack of information. We found that the two QTLs for flowering time were not located near the antibiosis QTLs (Figure 2). This leads to two conclusions, one being that the late-flowering habit of the Himeshirazu cultivar might not be an obstacle to the breeding of elite pest-resistant cultivars and the other conclusion is that the deferral of the plant developmental stage might not be a direct cause of antibiosis resistance. Since the major genes for flowering time do not lie near the antibiosis QTLs, it should be easy to produce mid- or early-maturing pest-resistant cultivars to fit the conventional cultivation system in Japan.

None of the QTLs detected in this study overlapped with the QTLs for antibiosis resistance reported by Komatsu et al. (2005). Thus, pubescence density and maturity are not the main cause of antibiosis resistance. However, we should not ignore the influence of pubescence or 
maturity when we investigate the immediate causes of pest resistance in the broad sense. Differences in the shape and posture of the pubescent hairs of soybean have been reported (Bernard and Singh, 1969; Palmer and Kilen, 1987) and we observed differences in the length and thickness of hairs in our materials but could not investigate the influence of these differences on antibiosis resistance. It was also unfeasible to investigate directly the relationship of pubescence density and antixenosis resistance in this study. In addition, the QTLs for antibiosis resistance explain in total $44 \%$ of phenotypic variance related to antibiosis (Komatsu et al., 2005), so it is likely that other genes for antibiosis lie unknown. From the results of this study, it is impossible to conclude that the QTLs for pubescence or maturity are not identical to the unknown gene for antibiosis resistance.

\section{Acknowledgments}

We are grateful to Dr. Kyuya Harada at Chiba University Japan for his original SSR loci information. We thank Dr. Takashi Wada, who kindly let us use the stereomicroscope with the image processor. We also thank Dr. Katsuhiro Matsui for his valuable advice and critical reading of the manuscript. This work was supported by a grant from the Ministry of Agriculture, Forestry and Fisheries of Japan (Green Technology Project DM-1204).

\section{References}

Bernard RL (1975) The inheritance of semi-sparse pubescence. Soybean Genet News1 2:33-34.

Bernard RL and Singh BB (1969) Inheritance of pubescence type in soybeans: Glabrous, curly, dense, sparse, and puberulent. Crop Sci 9:192-197.

Bonato ER and Vello NA (1999) E6, a dominant gene conditioning early flowering and maturity in soybeans. Genet Mol Biol 22:229-232.

Carpentieri-Pípolo V, Almeida LA, Kiihl RAS and Rosolem CA (2000) Inheritance of long juvenile period under short day conditions for the BR80-6778 soybean (Glycine max (L.) Merrill) line. Euphytica 112:203-209.

Churchill GA and Doerge RW (1994) Empirical threshold values for quantitative trait mapping. Genetics 138:963-971.

Cregan PB, Jalvik T, Bush AL, Shoemaker RC, Lark KG, Kahler AL, Kaya N, VanToai TT, Lohnes DG, Chung J, et al. (1999) An integrated genetic linkage map of the soybean genome. Crop Sci 39:1464-1490.

Doyle JJ and Doyle JL (1990) Isolation of plant DNA from fresh tissue. Focus 12:13-15.

Funatsuki K, Kawaguchi K, Matsuba S, Sato Y and Ishimoto M (2005) Mapping of QTL associated with chilling tolerance during reproductive growth in soybean. Theor Appl Genet 111:851-861.
Harada K and Xia Z (2004) Soybean genomics: Efforts to reveal the complex genome. Breed Sci 54:215-224.

Kogan M and Ortman EE (1978) Antixenosis - A new term proposed to replace Painter's 'nonpreference' modality of resistance. Bull Entomol Soc Am 24:175-176.

Komatsu K, Okuda S, Takahashi M and Matsunaga R (2004) Antibiotic effect of insect-resistant soybean on common cutworm (Spodoptera litura) and its inheritance. Breed Sci 54:27-32.

Komatsu K, Okuda S, Takahashi M, Matsunaga R and Nakazawa Y (2005) QTL mapping of antibiosis resistance to common cutworm (Spodoptera litura Fabricius) in soybean. Crop Sci 45:2044-2048.

Lambert L, Beach RM, Kilen TC and Todd JW (1992) Soybean pubescence and its influence on larval development and oviposition preference of lepidopterous insects. Crop Sci 32:463-466.

Lander ES, Green P, Abrahamson J, Barlow A, Daly MJ, Lincoln SE and Newberg L (1987) Mapmaker: An interactive computer package for constructing genetic linkage maps of experimental and natural populations. Genomics 1:174-181.

Molnar SJ, Rai S, Charette M and Cober ER (2003) Simple sequence repeat (SSR) markers linked to $E 1, E 3, E 4$, and $E 7$ maturity genes in soybean. Genome 46:1024-1036.

Nault BA, All JN and Boerma HR (1992) Resistance in vegetative and reproductive stages of a soybean breeding line to three defoliating pests (Lepidoptera, Noctuidae). J Econ Entomol $85: 1507-1515$.

Palmer RG and Kilen TC (1987) Qualitative genetics and cytogenetics. In: Wilcox JR (ed) Soybeans: Improvement, Production, and Uses (Agronomy 16). 2nd edition. American Society of Agronomy, Madison, pp 135-209.

Ray JD, Hinson K, Mankono JEB and Malo MF (1995) Genetic control of a long-juvenile trait in soybean. Crop Sci 35:1001-1006.

Rector BG, All JN, Parrott WA and Boerma HR (1998) Identification of molecular markers linked to quantitative trait loci for soybean resistance to corn earworm. Theor Appl Genet 96:786-790.

Rector BG, All JN, Parrott WA and Boerma HR (1999) Quantitative trait loci for antixenosis resistance to corn earworm in soybean. Crop Sci 39:531-538.

Reynolds GW and Smith CM (1985) Effects of leaf position, leaf wounding, and plant age of two soybean genotypes on soybean looper (Lepidoptera, Noctuidae) growth. Environ Entomol 14:475-478.

Woodworth C M (1921) Inheritance of cotyledon, seed-coat, hilum, and pubescence colors in soybeans. Genetics 6:487553.

Zeng ZB (1993) Theoretical basis for separation of multiple linked gene effects in mapping quantitative trait loci. Proc Natl Acad Sci USA 90:10972-10976.

Zeng ZB (1994) Precision mapping of quantitative trait loci. Genetics 136:1457-1468.

Associate Editor: Everaldo Gonçalves de Barros 